\title{
Thermal Nieh-Yan anomaly in Weyl superfluids
}

\author{
J. Nissinen $\oplus^{1, *}$ and G. E. Volovik ${ }^{1,2, \dagger}$ \\ ${ }^{1}$ Low Temperature Laboratory, Department of Applied Physics, Aalto University, FI-00076 AALTO, Finland \\ ${ }^{2}$ Landau Institute for Theoretical Physics, 142432 Chernogolovka, Russia
}

(Received 28 May 2020; revised 17 July 2020; accepted 20 July 2020; published 19 August 2020)

\begin{abstract}
We discuss the possibility of torsional Nieh-Yan anomaly of the type $\partial_{\mu}\left(e j_{5}^{\mu}\right)=\gamma T^{2}\left(\mathcal{T}^{a} \wedge \mathcal{T}_{a}\right)$ in Weyl superfluids, where $T$ is the infrared (IR) temperature scale and $\mathcal{T}^{a}$ is the effective or emergent torsion from the superfluid order parameter. As distinct from the dimensionful ultraviolet (UV) parameter $\Lambda^{2}$ in the conventional torsional Nieh-Yan anomaly, the parameter $\gamma$ is dimensionless in canonical units. This suggests that such dimensionless parameter may be fundamental, being determined by the geometry, topology, and number of chiral quantum fields in the system. By comparing this to a Weyl superfluid with low-temperature corrections, $T \ll \Delta_{0}$, we show that such a term does exist in the hydrodynamics of a chiral $p$-wave superfluid, such as ${ }^{3} \mathrm{He}-\mathrm{A}$, or a chiral superconductor. We also discuss and show how other $T^{2}$ terms of similar form and of the same order in gradients, coming from, e.g., Fermi-liquid corrections, effective curvature, and the chiral chemical potential, can also be expressed in terms of dimensionless fundamental parameters with emergent low-energy relativistic fields. Lastly, we discuss our results in comparison to relativistic Weyl fermions and the connection of the torsional gravitational anomalies to thermal transport in Weyl systems.
\end{abstract}

DOI: 10.1103/PhysRevResearch.2.033269

\section{INTRODUCTION}

In nonrelativistic topological matter, effectively quasirelativistic description of low-energy quasiparticles with linear spectrum phenomena may emerge $[1,2]$. In particular, in three spatial dimensions at a generic (twofold) degenerate fermion band crossing at momentum $\mathbf{p}_{W}$, the Hamiltonian is of the Weyl form [1,3-5]

$$
H_{W}=\sigma^{a} e_{a}^{i}\left(p-p_{W}\right)_{i}+\cdots,
$$

where the $e_{a}^{i}=\left.\partial_{p_{i}} H(\mathbf{p})\right|_{p_{W}}$ are the linear coefficients of the Hermitean Pauli matrices $\sigma^{a}, a=1,2$, and 3, in a Taylor expansion close to the Weyl node(s) at $\mathbf{p}_{W}$. The net chirality $\sum_{\left\{\mathbf{p}_{W}\right\}} \operatorname{sgn}\left(\operatorname{det} e_{a}^{i}\right)$ vanishes in the system [5]. For slowly varying parameters in the operator $\sigma^{a} e_{a}^{\mu} i \partial_{\mu} \equiv i \partial_{t}-H_{W}$, the semiclassical fields $e_{a}^{\mu}(x)=\left\{e_{a}^{t}, e_{a}^{i}\right\}$ are promoted to background space-time tetrad fields with dimensions of unity for temporal indices $e_{a}^{t}$ and velocity for spatial $e_{a}^{i}$. At the level of the Hamiltonian, the shift of the Weyl node $\mathbf{p}_{W}$ acts formally as an emergent (axial) gauge field with emergent Lorentz symmetry to the linear order. However, the Taylor expansion (1) at $\mathbf{p}_{W}$ is valid at much lower scales $\ll p_{W}$. If the fermions are charged, they can in addition couple to the electromagnetic vector potential via minimal coupling.

\footnotetext{
*jaakko.nissinen@aalto.fi

† grigori.volovik@aalto.fi
}

Published by the American Physical Society under the terms of the Creative Commons Attribution 4.0 International license. Further distribution of this work must maintain attribution to the author(s) and the published article's title, journal citation, and DOI.
These background fields imply the chiral anomaly for the low-energy massless quasiparticles. For the applications of the chiral anomaly in Weyl semimetal and Weyl superfluids/superconductors, see, e.g., Refs. [1,5-7]. In particular, the nontrivial coordinate dependence (torsion) related to the tetrads $e_{a}^{\mu}(x)$ in (1) can lead to the gravitational NiehYan (NY) anomaly [8-18]. Nevertheless, the relativistic NY contribution to the anomaly has remained contentious and subtle due to presence of a dimensionful ultraviolet (UV) scale $\Lambda^{2}$ with canonical dimensions of momentum, as required by the canonical dimensions of $e_{\mu}^{a}$. In condensed matter, the simplest way to understand this nonuniversal cutoff is the dependence of the expansion (1) on microscopics, see Ref. [16] for an example with an explicit $\Lambda$ provided by the nonrelativistic Fermi-liquid UV completion, and the discussion below.

Here we discuss the temperature corrections to the gravitational NY anomaly, such temperature corrections being absent for U(1) gauge fields in the chiral anomaly. We then compare the anomaly current to finite-temperature terms in the hydrodynamic momentum transport of chiral Weyl superfluids at low temperature. The results apply for the chiral superconductors as well, when the electromagnetic potential is added $\mathbf{v}_{s} \rightarrow \mathbf{v}_{s}-e \mathbf{A} / m$ in some convenient gauge. We show that the momentum current has a term originating from the torsional NY anomaly, among various other temperature terms of the same order but different origin in the free energy. All such second order $T^{2}$ corrections in the low-temperature expansion of the free energy take an effectively relativistic form. Their dimensionless prefactors do not depend on the details of the microscopic physics and, instead, are determined by geometry, topology, and the number of effective fermionic species $[1,19]$. Specifically, we compare the torsional finitetemperature corrections to the lowest order gradient terms 
in the free energy of the chiral superfluid, and identify the contribution from the chiral NY anomaly in the low-energy quasirelativistic Weyl superfluid. This leads us to conjecture that the finite temperature IR-scale NY anomaly term can be similarly universal in general Weyl systems. Finally, we compare these results to relativistic Weyl fermions with positive and negative branches at zero momentum.

\section{TORSIONAL ANOMALY}

For space-times with torsion (and curvature), Nieh and Yan [8-10] introduced the four-dimensional invariant

$$
N=\mathcal{T}^{a} \wedge \mathcal{T}_{a}-e^{a} \wedge e^{b} \wedge R_{a b},
$$

where $e^{a}=e_{\mu}^{a} d x^{\mu}$ is the local tetrad 1-form field and $\mathcal{T}^{a}=$ $d e^{a}+\omega^{a}{ }_{b} \wedge e^{b}$ and $R_{b}^{a}=d \omega_{b}^{a}+\omega^{a}{ }_{c} \wedge \omega_{b}^{c}$, in terms of the tetrad and spin connection $\omega_{b}^{a}=\omega_{\mu b}^{a} d x^{\mu}$. As usual, the spacetime metric follows as $g_{\mu \nu}=e_{\mu}^{a} e_{\nu}^{b} \eta_{a b}$, where $\eta_{a b}$ is the local orthonormal (Lorentz) metric. This invariant can be written, using the associated Bianchi identities, as

$$
N=d Q, Q=e^{a} \wedge \mathcal{T}_{a} .
$$

$N$ is a locally exact 4-from independent from $\operatorname{tr}(R \wedge R)$ and the dual of the scalar curvature $\sqrt{g} \mathcal{R}$ in the presence of nonzero torsion. It can be associated with a difference of two topological terms, albeit in terms of an embedding to five dimensions [13-15]. In terms of four-dimensional chiral fermions on such a space-time, it has been suggested that this invariant contributes to the axial anomaly, i.e., the anomalous production of the chiral current:

$$
\partial_{\mu}\left(e j_{5}^{\mu}\right)=\frac{\Lambda^{2}}{4 \pi^{2}} N(\mathbf{r}, t),
$$

where $e=\operatorname{det} e_{\mu}^{a}$ and $e j_{5}^{\mu}$ is the axial current (pseudotensor) density and the nonuniversal parameter $\Lambda$ has dimension of relativistic momentum (mass) $[\Lambda]=[1 / L]=[M]$ and is determined by some ultraviolet (UV) energy scale.

Given the relativistic anomaly term (4), there has been several attempts to consider the Nieh-Yan anomaly in condensed matter systems with Weyl fermions like (1), see e.g. [16-18,20]. However, in relativistic systems, the high-energy cutoff $\Lambda$ is not a well defined parameter and, moreover, can be in addition anisotropic in condensed matter systems. There the complete UV theory is, of course, non-Lorentz invariant and the linear, quasirelativistic Weyl regime is valid at much lower scales where (1) applies. Moreover, the anomalous hydrodynamics of superfluid ${ }^{3} \mathrm{He}$ at zero temperature suggests that the chiral anomaly is either completely exhausted by the emergent axial gauge field corresponding to the shift of the node or, conversely, by the gravitational NY anomaly term arising from the tetrad and spin-connection for local combined orbitalgauge invariance along the uniaxial symmetry direction. This was recently shown in Ref. [16], as well as that the low-energy theory satisfies the symmetries and conservations laws related to an emergent quasirelativistic space-time with torsion, $\Lambda$ being determined from the UV-scale where the linear Weyl approximation breaks down as dictated by the underlying $p$-wave BCS Fermi superfluid. Here we will consider the temperature corrections to the anomaly and the hydrodynamic free-energy in the chiral Weyl superfluid, with the expectation that $\Lambda=T, k_{B}=1$, with some dimensionless prefactor $\gamma$, in units where the tetrads have canonical dimensions of velocity, or the relevant "speed of light" is set unity. Notably, the IR $T^{2}$-correction to the anomaly would be absent for U(1) gauge fields.

In terms of effective low-energy actions, the fully relativistic analogs work unambiguously only for terms in the effective action with dimensionless coefficients. Perhaps the most well-known example being the 2+1-dimensional topological Chern-Simons (CS) terms describing the quantum Hall effect, see, however, Ref. [21]. Gravitational ChernSimons terms can be similarly quantized in terms of chiral central charge which has relation to thermal transport and the boundary conformal field theory $[22,23]$. The CS action was recently generalized to $3+1 \mathrm{~d}$ (and higher odd space dimensions) crystalline topological insulators, using so-called dimensionful elasticity tetrads $E$ with dimension $[E]=[1 / L]=$ $[M]$. Topological polarization terms can also be written down [24] by dimensional descent. The ensuing higher dimensional Chern-Simons and polarization terms are expressed as the mixed responses $E \wedge A \wedge d A$ and $E \wedge E \wedge d A$ with quantized dimensionless coefficients [24-26].

Another such example is the temperature correction to curvature effects, with $\delta S_{\text {eff }}=\int T^{2} \mathcal{R}$ in the low-energy action [19]. This represents the analog of the gravitational coupling (inverse Newton constant) in the low-energy action, where $\mathcal{R}$ is the effective scalar space-time curvature of the medium felt by the quasiparticles. Since $[T]^{2}[\mathcal{R}]=[M]^{4}$, the coefficient of this term is dimensionless, and can be given in terms of universal constants: it is fully determined by the number of the fermionic and bosonic species in the effective theory on flat background, and thus works both in relativistic and nonrelativistic systems [19]. The same universal behavior takes place with the terms describing the chiral magnetic and chiral vortical effects in Weyl superfluid ${ }^{3} \mathrm{He}-\mathrm{A}$, where the coefficients are dimensionless [1,27,28]. Similarly, it has been observed that the coefficient of the $\operatorname{tr}(R \wedge R)$ gravitational anomaly in chiral Weyl systems affects the thermal transport coefficients in flat space [29-35]. These coefficients are fundamental, being determined by the underlying degrees of freedom in addition to symmetry, topology and geometry. From this perspective especially, since the NY form is second order in derivatives and can be computed from linear response on flat space, our findings are very interesting and warrant further research.

Our goal in the present paper is to separate different $T^{2}$ contributions in the hydrodynamic free energy of Weyl superfluids in order to identify the terms responsible for different relativistic phenomena, including a term from the thermal Nieh-Yan anomaly in the current, as well as similar gravitational terms corresponding to the scalar curvature and chemical potential, $\int T^{2}\left(\mathcal{R}+\mu^{2}\right)$, where $\mu$ is the chiral chemical potential $\mu \ll T$.

\section{TEMPERATURE CORRECTION TO THE NIEH-YAN TERM}

The relativistic zero-temperature anomaly term in the axial current production $\Lambda^{2}\left(\mathcal{T}^{a} \wedge \mathcal{T}_{a}+e^{a} \wedge e^{b} \wedge R_{a b}\right)$ is still 
not confirmed in general, see however Refs. [16,36] for condensed matter Weyl fermions. On one hand, the UV cutoff parameter $\Lambda$ is not well-defined in relativistic field theory with fundamental chiral fermions. On the other hand, such a cutoff is not in general available in nonrelativistic matter with quasirelativistic low-energy chiral fermions and can be anisotropic [16] or even zero. However, a term of the form $\gamma T^{2}\left(\mathcal{T}^{a} \wedge \mathcal{T}_{a}+e^{a} \wedge e^{b} \wedge R_{a b}\right)$ has the proper dimensionality $[M]^{4}$, and its prefactor $\gamma$ could be a universal constant in canonical units, being expressed via some invariant related to the low-energy degrees of freedom.

For concreteness, we focus on the finite temperature NiehYan anomaly in chiral $p$-wave Weyl superfluid (such as ${ }^{3} \mathrm{He}$ A) with the expectation that

$$
\partial_{\mu}\left(e j_{5}^{\mu}\right)=\gamma T^{2} N(\mathbf{r}, t),
$$

and check whether the dimensionless parameter $\gamma$ can be universal. We now use the result obtained by Khaidukov and Zubkov [37] and Imaki and Yamamoto [38] for the finite temperature contribution to the chiral current from the chiral vortical effect. For a single (complex) chiral fermion, one has for the chiral current

$$
j_{5}^{k}=-\frac{T^{2}}{24} \epsilon^{k i j} \mathcal{T}_{i j}^{0} .
$$

We assume that this current can be covariantly generalized to the 4-current:

$$
e j_{5}^{\mu}=-\frac{T^{2}}{24} \epsilon^{\mu \nu \alpha \beta} e_{\nu a} \mathcal{T}_{\alpha \beta}^{a} .
$$

Then one obtains the divergence

$$
\partial_{\mu}\left(e j_{5}^{\mu}\right)=-\frac{T^{2}}{48} \epsilon^{\mu \nu \alpha \beta} \mathcal{T}_{a \mu \nu} \mathcal{T}_{\alpha \beta}^{a} .
$$

In the presence of curvature $R(\omega)$, this becomes the temperature correction to the full Nieh-Yan term in Eq. (5), where now the nonuniversal cutoff $\Lambda$ is substituted by the well defined IR temperature scale $T$, and the dimensionless parameter $\gamma=$ 1/12:

$$
\partial_{\mu}\left(e j_{5}^{\mu}\right)=-\frac{T^{2}}{12} N(\mathbf{r}, t) .
$$

Note that it is possible that the local relativistic (Tolman) temperature $T=T_{0} /\left|e_{t}^{0}\right|$ enters the local anomaly, while the constant $T_{0}$ is the global equilibrium temperature of the condensed matter system [19]. In (1), we have simply $e_{t}^{0}=-1$.

\section{FROM RELATIVISTIC PHYSICS TO CHIRAL WEYL SUPERFLUID}

In the presence of a finite Weyl node $p_{W} \neq 0$, the chiral anomaly for the chiral current leads to the anomalous production of the linear momentum $[1,16,36]$. Even though chiral current is not well-defined at high-energy, the spectral flow of chiral quasiparticles is accompanied by the spectral flow of the linear momentum $\mathbf{p}_{W}$ of the Weyl point. In ${ }^{3} \mathrm{He}-\mathrm{A}$, there are two (spin-degenerate) Weyl points with opposite chirality and opposite momenta, $\mathbf{p}_{W \pm}= \pm p_{F} \hat{\mathbf{l}}$, where $\hat{\mathbf{l}}$ is the unit vector of the orbital angular momentum of the superfluid. In particular, the anomalous production of the linear momentum density $\mp p_{F} n_{ \pm}$at nodes of the two opposite Weyl points sum-up leading to

$$
\dot{\mathbf{P}}_{\text {anom }}=-p_{F} \hat{\mathbf{l}}\left(\partial_{t} n_{5}\right)
$$

where $n_{5}=n_{+}-n_{-}$is the chiral density. The corresponding quasirelativistic momentum density of the Weyl fermions, valid in the vicinity of the node, is [16]

$$
\mathbf{P}_{\mathrm{NY} \text {-node }}=-p_{F} \hat{\mathbf{l}} e j_{5}^{0} .
$$

Thus Eq. (8) gives the temperature correction to this anomalous momentum production, leading to a mass current due to thermal NY anomaly. Next we discuss this in detail for the quasiparticles and the superfluid vacuum in the nonrelativistic chiral Weyl $p$-wave superfluid, using a Landau level model for the currents [16,39]. For relativistic Weyl fermions, see $[38,40]$. For considerations of similar temperature effects in chiral Weyl superfluid, see Ref. [41].

\section{A. Nieh-Yan term from the hydrodynamics of chiral Weyl superfluid}

It is known that the hydrodynamics of chiral gapless ${ }^{3} \mathrm{He}-\mathrm{A}$ experiences momentum anomalies related to the spectral flow through the Weyl points. Let us express Eqs. (10) and (11) in terms of the hydrodynamic variables and quasiparticles of the chiral superfluid. The Weyl fermions arise from the BdG Hamiltonian close to the nodes,

$$
H_{\mathrm{BdG}}(-i \partial)=\left(\begin{array}{cc}
\epsilon(-i \partial) & \frac{1}{2 i}\left\{\partial_{i}, \Delta^{i}\right\} \\
\frac{1}{2 i}\left\{\partial_{i}, \Delta^{* i}\right\} & -\epsilon(i \partial)
\end{array}\right) .
$$

Here, $\epsilon(\mathbf{p})=\frac{p^{2}-p_{F}^{2}}{2 m}$ is the normal state dispersion minus the Fermi level $\mu_{F} ; \Delta_{i}=c_{\perp}(\hat{\mathbf{m}}+i \hat{\mathbf{n}})$ is the order parameter of the $p+i p$ chiral superfluid; $\hat{\mathbf{l}}=\hat{\mathbf{m}} \times \hat{\mathbf{n}}$ is the unit vector in the direction of the orbital angular momentum of Cooper pairs. The anisotropic node velocities are $c_{\|}=v_{F}$ and $c_{\perp}=\Delta_{0} / p_{F}$, constituting effective speeds of light in Weyl equation along and transverse to $\hat{\mathbf{l}}$, respectively. In the weak coupling BCS theory, $c_{\perp} \ll c_{\|}$, and in ${ }^{3} \mathrm{He}-\mathrm{A}$, their ratio is of order $10^{-3}$. In a chiral superconductor, we in addition perform the minimal coupling $\epsilon\left(-i \partial_{i}\right) \rightarrow \epsilon\left(i D_{i}\right)+e A_{0}$, where $D_{i}=\partial_{i}-e A_{i}$ is the gauge covariant derivative and $A_{\mu}$ is the electromagnetic potential. Equivalently this amounts to $\mathbf{v}_{\mathbf{s}} \rightarrow \mathbf{v}_{s}-\frac{e \mathbf{A}}{m}$ in the free-energy. In what follows, we ignore the superfluid velocity (giving rise to spin-connection in addition to tetrads) in the anomaly considerations. Then the only hydrodynamic variable appearing in the torsion is the unit vector of the orbital momentum î [16]. Our results can afterwards then generalized to include the superfluid velocity appearing in the free energy, with the expectation that curvature term is included in the NY form as in Eqs. (2), (5), and as long as we enforce proper normalization due to momentum space anisotropy of thermal fluctuations, see Appendix A 2 [16]. See also Ref. [42] for two-dimensional finite-temperature effects of curvature without torsion.

The Weyl nodes are at $\mathbf{p}_{W \pm}= \pm p_{F} \hat{\mathbf{l}}$. Expanding the Hamiltonian as $H_{\mathrm{BdG}} \simeq \sigma^{a} e_{a}^{i}\left(\hat{p}-p_{W}\right)_{i}$, the vierbein $\mathbf{e}_{a}^{i}$ in the 
vicinity of the Weyl point take the form

$$
e_{a}^{\mu}=\left\{e_{a}^{t}, \mathbf{e}_{a}^{i}\right\}=\left(\begin{array}{cc}
1 & 0 \\
0 & c_{\perp} \hat{\mathbf{m}} \\
0 & c_{\perp} \hat{\mathbf{n}} \\
0 & c_{\|} \hat{\mathbf{l}}
\end{array}\right), \quad a=0,1,2,3 .
$$

For the inverse vierbein $\mathbf{e}_{a}^{i} \mathbf{e}_{j}^{a}=\delta_{j}^{i}$, we have

$$
e_{\mu}^{a}=\left\{e_{t}^{a}, \mathbf{e}_{i}^{a}\right\}=\left(\begin{array}{cccc}
1 & 0 & 0 & 0 \\
0 & \frac{1}{c_{\perp}} \hat{\mathbf{m}} & \frac{1}{c_{\perp}} \hat{\mathbf{n}} & \frac{1}{c_{\|}} \hat{\mathbf{l}}
\end{array}\right) .
$$

In order to compute the thermal contribution from the quasiparticles in the presence of torsion, we assume that the tetrad gives rise to a constant torsion via $\hat{\mathbf{m}}=\hat{\mathbf{x}}, \hat{\mathbf{n}}=\hat{\mathbf{y}}-$ $T_{B} x \hat{\mathbf{z}}$, where $T_{B} \ll 1$ is a perturbation [16,36,39]. In this case, the three-dimensional spectrum organizes into onedimensional states on two-dimensional Landau levels (LLs) $[1,17,36,39,40]$. See also e.g. $[43,44]$ for reviews of vector and axial U(1) fields in semimetals. In our case, the relevant spatial torsion is

$$
\frac{1}{2} \epsilon^{i j k} \mathcal{T}_{j k}^{3} e_{3}^{i}=c_{\|} \hat{\mathbf{l}} \cdot\left(\nabla \times \frac{\hat{\mathbf{1}}}{c_{\|}}\right) \equiv T_{B},
$$

and $p_{z} T_{B}=p_{z} \hat{\mathbf{l}} \cdot \nabla \times \hat{\mathbf{I}}=p_{z} \mathcal{T}_{x y}^{z}$ is playing the role of torsional effective magnetic field with momentum charge. For more on LL spectrum in ${ }^{3} \mathrm{He}-\mathrm{A}$ and the nonrelativistic anisotropic model with torsion, see Appendix A 2 and Refs. [16,36,39,45].

The anomalous (chiral) quasiparticle current along $\hat{\mathbf{l}} \approx \hat{\mathbf{z}}$ becomes

$$
\begin{aligned}
\mathbf{P}^{\mathrm{qp}} \cdot \hat{\mathbf{I}} & =-2 \int_{0}^{\infty} N_{\mathrm{LL}}\left(p_{z}\right) d p_{z} p_{z} n_{F}\left(\epsilon_{p_{z}}-\mu_{F}\right) \\
& =-\left(\frac{p_{F}^{3}}{6 \pi^{2}}+\frac{p_{F} T^{2}}{6 c_{\perp}^{2}}\right) \hat{\mathbf{I}} \cdot \nabla \times \hat{\mathbf{I}} \\
& =\mathbf{j}_{\mathrm{anom} \|}^{\mathrm{vac}}+\mathbf{j}_{\text {anom } \|}^{\mathrm{qp}}(T),
\end{aligned}
$$

where $n_{F}(x)=\left(e^{\beta x}+1\right)^{-1}$ is the quasiparticle distribution function and a factor of two comes from the spin-degeneracy. In order to compute the integral, we have used $T \ll \Delta_{0} \ll$ $\mu_{F}$ as well as the linear expansion with anisotropic momentum scaling, where close to the nodes thermal fluctuations are $\Delta p_{\perp} \sim \frac{T}{\Delta_{0}} p_{F}=\frac{T}{c_{\perp}}, \Delta p_{\|}=\left(\frac{c_{\perp}}{c_{\|}}\right) \frac{T}{c_{\perp}}$ for both $T, \epsilon \ll m c_{\perp}^{2}$. This is due to the anisotropy of the linear Weyl regime in ${ }^{3} \mathrm{He}$ A with thermal fluctuations $\epsilon \sim T / 2$. See Appendixes A 1, A 2, and Refs. [16,36] for more details. We conclude that $\mathbf{j}_{\text {anom }}^{\mathrm{vac}}$ is the anomalous superfluid current from filled quasiparticle states, see Eq. (21) below,

$$
\mathbf{j}_{\text {anom } \|}^{\mathrm{vac}}=-\frac{p_{F}^{3}}{6 \pi^{2}} \hat{\mathbf{l}}(\hat{\mathbf{l}} \cdot \nabla \times \hat{\mathbf{l}})=-\frac{C_{0}}{2} \hat{\mathbf{l}}(\hat{\mathbf{l}} \cdot \nabla \times \hat{\mathbf{l}}),
$$

whereas the finite temperature contribution to the quasiparticle momentum is

$$
\mathbf{j}_{\text {anom } \|}^{\mathrm{qp}}(T)=-\frac{p_{F} T^{2}}{6 c_{\perp}^{2}} \hat{\mathbf{l}}(\hat{\mathbf{l}} \cdot \nabla \times \hat{\mathbf{l}}) .
$$

and arises due to thermal normal component close to the nodes. These contributions to the anomalous vacuum current from the perspective of the superfluid are analyzed in the next subsection.
In addition, the relativistic anomaly results in Sec. III, with Eq. (7), and the Landau level argument for the chiral superfluid suggest the following temperature correction to the anomalous momentum in ${ }^{3} \mathrm{He}-\mathrm{A}$, in the vicinity of the node, from the thermal Nieh-Yan anomaly [16]:

$$
\mathbf{P}_{\text {NY-node }}(T)=-p_{F} \hat{\mathbf{l}} n_{5}(T)=\frac{p_{F} T^{2}}{12 c_{\perp}^{2}} \hat{\mathbf{l}}(\hat{\mathbf{l}} \cdot(\nabla \times \hat{\mathbf{l}})) .
$$

The quasiparticle density $n_{5}(T)$ between the two Weyl nodes is computed in the LL model of Appendix A 2 from a similar integral as Eq. (16),

$$
\begin{aligned}
n_{5}(T) & =-2 \int_{0}^{\infty} N_{\mathrm{LL}}\left(p_{z}\right) d p_{z}\left[n_{F}\left(\epsilon_{p_{z}}-\mu_{F}\right)-\Theta\left(\mu-\epsilon_{p_{z}}\right)\right] \\
& =\frac{-T_{B}}{2 \pi^{2}} \int_{0}^{\infty} d x x n_{F}(x)=\frac{-T^{2}}{12 c_{\perp}^{2}}(\hat{\mathbf{l}} \cdot \nabla \times \hat{\mathbf{l}}) .
\end{aligned}
$$

As for the total current, this is the contribution from the linear spectrum close to the nodes due to thermal fluctuations, with $T$ playing the role of the cutoff relevant for the full dispersion of the chiral superfluid, such as ${ }^{3} \mathrm{He}-\mathrm{A}$. The thermal fluctuations for the superfluid are discussed in Appendix A 1.

What have been calculated above are actually the temperature dependence of the anomalous torsional response of the Weyl superfluid, i.e., the chiral momentum-density and number densities in response to $T_{B}$, see, e.g., Ref. [32]. Note in particular that the leading torsional contribution from the NY anomaly in Eqs. (16) and (20) from thermal fluctuations is suppressed by the factor $c_{\perp}^{-2}$ as was found as the leading order term to Eq. (2) in Ref. [16] including the curvature contribution to the anomaly. This is in contrast to the subleading torsion part $S=\frac{1}{2} \epsilon^{i j k} e_{i}^{3} \mathcal{T}_{j k}^{3}=\frac{T_{B}}{c_{\|}^{2}}$ from (14) that would arise in the LL model without the anisotropic thermal fluctuations discussed. Apart from such geometric factors, it is amusing to see that the nonrelativistic thermal integrals in Eqs. (16) and (20) with explicit nonrelativistic vacuum regulatization due to the filled quasiparticle states coincide with similar expression for relativistic Weyl fermions with positive and negative branches [28,30-32,37,38], see Appendix A 3. This is by the universality of thermal fluctuations close to the node and the underlying reason why the corresponding anomaly contribution can be universal.

\section{B. Anomalous vacuum current}

Now we discuss the anomalous current from the underlying vacuum with torsion fields, i.e., the current in the chiral superfluid (or superconductor).

The hydrodynamic anomaly in momentum conservation arises between the quasiparticles and vacuum mass current $\mathbf{P}=\mathbf{j}$ of the superfluid, which at $T=0$ has the following general form $[1,46,47]$ :

$$
\mathbf{j}=\rho \mathbf{v}_{s}+\frac{1}{4 m} \nabla \times(\rho \hat{\mathbf{l}})-\frac{C_{0}}{2} \hat{\mathbf{l}}(\hat{\mathbf{l}} \cdot(\nabla \times \hat{\mathbf{l}})),
$$

where the last term is anomalous with the parameter $C_{0}$ from the combined orbital-gauge symmetry of the superfluid [48], that fully determines the axial anomaly in the system due to the Weyl quasiparticles of the superfluid [1]

$$
C_{0}(T=0)=p_{F}^{3} / 3 \pi^{2}=\rho,
$$


where we ignore corrections of the order of $\left(\Delta_{0}^{2} / E_{F}^{2}\right)=$ $\left(c_{\perp} / c_{\|}\right)^{2} \ll 1$ at zero temperature to the density from pairing $\left[\rho-C_{0} \sim \rho\left(\Delta_{0}^{2} / E_{F}^{2}\right)\right]$. Notably, this term exists only on the weak coupling side of the topological BEC-BCS Lifshitz transition, where the pair of the Weyl points with $\mathbf{p}_{W \pm}=$ $\pm p_{F} \hat{\mathbf{l}}$ appears in the quasiparticle spectrum $[1,48]$.

Here we are interested in the temperature correction to the anomaly, which corresponds to the Nieh-Yan term. The extension of the anomalous current to nonzero temperature gives

$$
\mathbf{P}_{\text {anom }}(T)=-\frac{1}{2} C_{0}(T) \hat{\mathbf{l}}(\hat{\mathbf{l}} \cdot(\nabla \times \hat{\mathbf{l}})),
$$

where according to Cross [46,47], the anomalous parameter $C_{0}(T)$ has the following temperature dependence at low $T \ll T_{c}$ :

$$
C_{0}(T)=C_{0}(0)-T^{2} \frac{p_{F}}{6 c_{\perp}^{2}}\left(1+\frac{m^{*}}{m}\right) .
$$

Here, $m^{*}$ is the effective mass of quasiparticles in the normal Fermi liquid, which differs from the bare mass $m$ of the ${ }^{3} \mathrm{He}$ atom due to the Fermi liquid corrections. In Eqs. (17) and (18), corresponding to the simple one-dimensional LL model, we have neglected the Fermi-liquid corrections due to interactions in the three-dimensional superfluid: An additional factor $\frac{1}{2}\left(\frac{m^{*}}{m}-1\right)\left(\rho_{n}^{(0)} / \rho\right)$ arises from the reduced quasiparticle momentum flow, due to Galilean invariance. The full current becomes [47]

$$
\mathbf{j}_{\text {anom }}=\frac{1}{1+\frac{1}{3} F_{1}^{s}\left(\rho_{n \|}^{(0)} / \rho\right)} \frac{m^{*}}{m} \mathbf{j}_{\text {anom }}^{(0)},
$$

where the Landau parameter $\frac{1}{3} F_{1}^{s}=\frac{m^{*}}{m}-1$ and $\rho_{n \|, \perp}^{(0)}(T)$ are the bare thermal quasiparticle densities without Fermi-liquid corrections, along and perpendicular to $\hat{\mathbf{l}}$, respectively. They are given by, see the Appendix A 1,

$$
\rho_{n \|}^{(0)}=\frac{\pi^{2} T^{2}}{\Delta_{0}^{2}} \rho, \quad \rho_{n \perp}^{(0)}=\frac{7 \pi^{4} T^{4}}{15 \Delta_{0}^{4}} \rho,
$$

where by Galilean invariance, $\rho \delta_{i j}=\rho_{s i j}+\rho_{n i j}$ at all temperatures. From Eqs. (25) and (26) we gather

$$
\frac{C_{0}(T)}{2}=\frac{p_{F}^{3}}{6 \pi^{2}}-\frac{p_{F} T^{2}}{6 c_{\perp}^{2}}-\frac{p_{F} T^{2}}{12 c_{\perp}^{2}}\left(\frac{m^{*}}{m}-1\right)+O\left(T^{4}\right),
$$

now with the finite temperature and Fermi-liquid corrections of Eq. (24) separated, corresponding to the result (18) when the Fermi-liquid corrections are ignored $m^{*} / m=1$.

We summarize these findings as follows. While Eq. (23) with temperature corrections Eq. (24) is not equal to the NY anomaly density $\mathbf{P}_{\mathrm{NY} \text {-node }}(T)$ Eq. (19), $\mathbf{P}_{\text {anom }}(T)$ represents the consistent anomaly [43] vacuum current from all filled states, which in addition depends on the nonfundamental parameters $p_{F}, m$, and $m^{*}$ via the Fermi-liquid corrections. At the same time, we expect that the $T^{2}$ contribution to the (covariant) NY anomaly arises from contributions close to the linear Weyl nodes and should contain fundamental and universal prefactors, as the axial quasiparticle density Eq. (20).

Concerning the anomalous vacuum momentum current of the superfluid (23), the reason for such parameters is that there are several different and interrelated $T^{2}$ contributions to the current and free energy in the chiral superfluid, and they correspond to hydrodynamics and phenomena with different origins and scales. Although of similar form in terms of the low-energy Goldstone variables of the superfluid, they can be expressed in relativistic form with fundamental parameters [19] only when carefully keeping track of each individual contribution to avoid double counting. In particular, in the next section we shall see that the nonfundamental parameters $m$ and $m^{*}$ do not enter the free energy or the final results, when expressed in terms of the correct relativistic variables valid in the quasirelativistic low-energy theory.

\section{RELATIVISTIC CORRECTIONS TO FREE-ENERGY}

Let us consider the $T^{2}$ corrections in the free energy which are second order in derivatives containing combinations of $\left(\hat{\mathbf{l}} \cdot \mathbf{v}_{s}\right)$ and $(\hat{\mathbf{l}} \cdot(\nabla \times \hat{\mathbf{l}}))$, neglecting all higher order $O\left(T^{4}, \partial^{3}\right)$ terms. These terms can be distributed into three groups, which have different dependence on $m^{*}$ and $m$ :

$$
\begin{gathered}
F=F_{1}+F_{2}+F_{3}, \\
F_{1}\left[\hat{\mathbf{l}}, \mathbf{v}_{s}\right]=\frac{p_{F}}{12} \frac{T^{2}}{c_{\perp}^{2}}\left(\hat{\mathbf{l}} \cdot \mathbf{v}_{s}\right)(\hat{\mathbf{l}} \cdot(\nabla \times \hat{\mathbf{l}})), \\
F_{2}\left[\hat{\mathbf{l}}, \mathbf{v}_{s}\right]=-\frac{p_{F} m^{*}}{96 m^{2}} \frac{T^{2}}{c_{\perp}^{2}}\left[4 m\left(\hat{\mathbf{l}} \cdot \mathbf{v}_{s}\right)-(\hat{\mathbf{l}} \cdot(\nabla \times \hat{\mathbf{l}}))\right]^{2}, \\
F_{3}[\hat{\mathbf{l}}]=-\frac{p_{F}}{288 m^{*}} \frac{T^{2}}{c_{\perp}^{2}} .
\end{gathered}
$$

The relativistic form of each free-energy contribution above arises separately as follows.

The term $F_{3}$ in Eq. (31) describes the universal temperature correction to the Newton gravitational coupling, which depends on the number of fermionic species [19]:

$$
F_{3}[\mathcal{R}, T]=-\frac{c_{\|}}{288} \frac{T^{2}}{c_{\perp}^{2}}(\hat{\mathbf{l}} \cdot(\nabla \times \hat{\mathbf{l}}))^{2}=\frac{T^{2}}{144} \sqrt{-g} \mathcal{R} .
$$

Note that being exressed in terms of the scalar curvature and metric determinant $\sqrt{-g}=1 / c_{\|} c_{\perp}^{2}$, this term becomes universal: it does not contain the microscopic parameters of the system: $p_{F}, m$, and $m^{*}$. The prefactor is fully determined by the number of fermionic species.

The term $F_{2}$ in Eq. (30) is expressed in terms of the combination $\hat{\mathbf{I}} \cdot \mathbf{v}=\hat{\mathbf{I}} \cdot \mathbf{v}_{s}-\frac{1}{4 m} \hat{\mathbf{l}} \cdot(\nabla \times \hat{\mathbf{l}})$, proportional to the ground state current which does not receive corrections due to Galilean invariance. Here the velocity $\mathbf{v}=\mathbf{j} / \rho$ is the velocity of the "total quantum vacuum", where $\mathbf{j} \equiv \mathbf{j}(T=0)$ is the total vacuum current in Eq. (21) at $T=0$. We conclude that the $F_{2}$ contribution gives the temperature $T$ and chemical potential $\mu$ correction to the free energy of the gas of chiral fermionic particles in the limit $|\mu| \ll T$ (see Eqs. (9.12) and (10.42) in Ref. [1]):

$$
\begin{aligned}
F_{2}[\mu, T] & =-\frac{p_{F} m^{*}}{96 m^{2}} \frac{T^{2}}{c_{\perp}^{2}}\left(4 m \hat{\mathbf{l}} \cdot \mathbf{v}_{s}-\hat{\mathbf{l}} \cdot(\nabla \times \hat{\mathbf{l}})\right)^{2} \\
& =-\frac{T^{2}}{6} \sqrt{-g} \mu^{2},
\end{aligned}
$$

where the chiral chemical potential of the superfluid is determined by the Doppler shift

$$
\mu_{R}=-\mu_{L}=p_{F} \mathbf{v} \cdot \hat{\mathbf{l}}=p_{F} \hat{\mathbf{l}} \cdot \mathbf{v}_{s}-\left(p_{F} / 4 m\right) \hat{\mathbf{I}} \cdot(\nabla \times \hat{\mathbf{l}}) .
$$


The final form of Eq. (33) does not contain microscopic parameters and also gives rise to the mass of "photon" in ${ }^{3} \mathrm{He}-\mathrm{A}$ [49]. We note that the current $\left.\frac{\delta F_{2}}{\delta \mathbf{v}_{s}}\right|_{\mathbf{v}_{s}=0}$ is the $m^{*} / m$ piece of the anomalous current (23) and Eq. (24). In principle, the chiral chemical potential may also serve as the $\Lambda$ parameter in the Nieh-Yan term when superflow and curvature terms are included at finite temperature, see however Appendix A 3 for the comparison of the LL model and relativistic fermions with chemical potential.

Finally $F_{1}$ in Eq. (29) is the term in the free energy without any factors of $m^{*} / m$, corresponding to the contribution from the consistent Nieh-Yan thermal anomaly in Eq. (18), with Fermi-liquid corrections Eq. (27),

$$
\begin{aligned}
\mathbf{P}_{\mathrm{anom}}(T) & =\frac{\delta F_{1}}{\delta \mathbf{v}_{s}}=\frac{p_{F}}{12} \frac{T^{2}}{c_{\perp}^{2}} \hat{\mathbf{l}}(\hat{\mathbf{l}} \cdot(\nabla \times \hat{\mathbf{l}})) \\
& \simeq-p_{F} n_{5}(T) \hat{\mathbf{l}},
\end{aligned}
$$

where the last formal equality was derived in Eq. (20). $\mathbf{P}_{\text {anom }}(T)$ is part of the total anomalous vacuum current with Fermi liquid corrections, whereas the second line is from the quasiparticle (momentum) density due to thermal fluctuations close to the node and is universal.

\section{CONCLUSIONS}

We discussed the possibility of thermal Nieh-Yan anomaly where the role of the nonuniversal dimensionful UV cutoff $\Lambda$ is played by the temperature IR scale, and the dimensionless prefactor $\gamma$ in the anomaly is universal. Such a contribution cannot arise for the chiral anomaly for U(1) gauge fields nor the conventional gravitational anomaly higher order in gradients, explicitly pertaining to the anomaly from torsion instead. In simple pragmatic terms, the temperature dependence results from the universal coupling of momentum to tetrads and the corresponding momentum dependence LL density of states.

We identified a contribution from this anomaly in the known low-temperature corrections of a nonrelativistic chiral $p$-wave Weyl superfluid (or superconductor). In this system $[1,16,36]$, the tetrad gravity and space-time with torsion arises as in, e.g., Refs. [50,51] from the Fermi condensate, while the anomaly results from thermal effects of the linear Weyl spectrum at finite momentum in the presence of an explicit vacuum of filled quasiparticles, although the end results are similar to relativistic fermions when interpreted carefully in terms of the anisotropy and cutoff of the quasirelativistic Weyl regime.

What we calculated, via the anisotropic Landau level model with nonrelativistic symmetries in Sec. IV, are actually the temperature dependence of the anomalous torsional conductivities $\sigma_{\mathcal{T}^{a}}$ of the quasiparticle system, i.e., the chiral momentum-density and number densities (at the nodes at finite momenta $\pm p_{F}$ ) in response to the spacelike torsion $c_{\|}^{-2} T_{B}=\frac{1}{2} \epsilon^{i j k} e_{i}^{3} \mathcal{T}_{j k}^{3}$. Namely, for example,

$$
\left\langle P^{a}\right\rangle=\left\langle e_{i}^{a} T^{0 i}\right\rangle=e_{i}^{a} \frac{\sigma_{\mathcal{T}^{b}}(T)}{2} \epsilon^{0 i j k} \mathcal{T}_{j k}^{b}=\delta^{a 3} \sigma_{T_{B}} T_{B},
$$

$P^{i}=T^{0 i}$, where $T^{\mu \nu}$ is the stress tensor, which are of second order in derivates and can be calculated in linear response, e.g., Kubo formulas, to the background tetrads [29,32,52,53].
A similar momentum anomaly in anisotropic system with nonrelativistic symmetries was also considered in Ref. [54]. For the zero-temperature case, see Refs. [16,36] as well as Refs. [40,55] for a relativistic model related to Weyl semimetals. We, however, stress that the universal gravitational NY anomaly and thermal physics we discussed arise in flat space from the geometric background fields in the low-energy quasiparticle Hamiltonian. These tetrads arise universally in all Weyl systems (1) and couple to the momentum [16,17,36,56] as in gravity. In particular, the torsional LL density of states is momentum dependent, similar to the (gravitational) response in the chiral vortical and magnetic effects, and leads to the finite temperature anomaly that does not exist for $U(1)$ gauge fields. The connection of our results and the relation of the gravitational NY anomaly, with the coefficient $\gamma$, to thermal transport in Weyl system should be further elucidated [29-31,33,35,41,57].

Detailed consideration of the temperature dependent anomaly terms in the hydrodynamics of the nonrelativistic $p$-wave chiral superfluid with quasirelativistic Weyl fermions demonstrates that in the hydrodynamics of this liquid there are several $T^{2}$ terms which can be assigned to different emergent relativistic phenomena, both anomalous and nonanomalous. In particular, we identified and discussed the term in the vacuum momentum corresponding to the (consistent) thermal Nieh-Yan anomaly [43]. This vacuum current depends on nonuniversal parameters with finite-temperature Fermiliquid corrections, but, as expected originates from universal anomalous thermal fluctuations close to the linear nodes with emergent quasirelativistic torsion with anisotropy. Note that in terms of the superfluid and Weyl fermions, the $T=0$ anomalous vacuum contribution to the current can be assigned only to a nonlocal action [58] which hints the existence of corresponding gravitational Wess-Zumino terms with torsion. We further showed how the various $T^{2}$ low-temperature corrections to the free energy can be grouped and written as lowenergy relativistic terms, including also chiral chemical potential and scalar curvature terms. They have dimensionless prefactors, which do not seem to depend on microscopic physics, but are fully determined by geometry, topology and the number of fermionic and bosonic quantum fields with thermal fluctuations. Detailed comparison of the finite temperature superfluid hydrodynamics with Fermi-liquid corrections to the anomalous quasiparticle axial current production, corresponding to the covariant torsional anomaly, in the presence of arbitrary textures and superfluid velocity remains to be identified [39,59]. See however Ref. [16] for the zero-temperature case.

Note added. After the initial submission of this paper as a preprint, arXiv:1909.08936v1, with the predicted $T^{2}$ contribution to the NY anomaly, Eqs. (9) and (35), Refs. [40,55,60] discussing related torsional anomaly phenomena at finite temperatures appeared. General aspects of the temperature anomaly in Weyl materials were further discussed in the short paper [61]. In particular, the result Eq. (9) has been confirmed in Ref. [40] by a direct calculation of the spectral flow of relativistic Landau levels in the presence of a constant torsional magnetic field $T_{\mu \nu}^{3}[17,39,45]$ at finite temperature. Here similar computations for the nonrelativistic Weyl superfluid in Eqs. (16) and (20) give corresponding results. While the current manuscript was being finalized, also the paper [62] 
appeared discussing the anomaly for relativistic fermion at finite temperature and chemical potential.

\section{ACKNOWLEDGMENTS}

G.E.V. thanks M. Zubkov for discussions. J.N. thanks Z.M. Huang for correspondence, T. Ojanen for discussions and S. Laurila for collaboration in Ref. [36]. This work has been supported by the European Research Council (ERC) under the European Union's Horizon 2020 research and innovation programme (Grant Agreement No. 694248).

\section{APPENDIX: VACUUM REGULARIZATION, ANISOTROPIC LANDAU LEVEL MODEL, AND THERMAL INTEGRALS}

In this Appendix, we shortly review the low-temperature corrections to the chiral superfluid and the quasirelativistic thermal integrals utilized in the main text. Although the temperature and Fermi-liquid corrections in Appendix A 1 chiral superfluid are well-known $[1,46,47]$, it is of interest to compare them to the chiral quasirelativistic Landau level model with torsion and explicit UV completion [16,36,39], Appendix A 2, as well to relativistic Weyl fermions with positive and negative branches [28,30,32], Appendix A 3. The last system has particles and antiparticles at finite chemical potential counted from the node and finite temperature with vacuum contributions subtracted, in contrast to nonrelativistic systems at finite chemical potential.

\section{Temperature corrections to normal and superfluid density in chiral superfluid}

We start with the corrections to the superfluid and normal density. The anisotropic chiral $p$-wave superfluid density is

$$
\rho_{n i j}^{(0)}=3 \rho \int d \Omega \hat{\mathbf{k}}_{i} \hat{\mathbf{k}}_{j} \int_{-\infty}^{\infty} d \epsilon\left(-\frac{\partial n_{F}\left(E_{\mathbf{k}}\right)}{\partial E_{\mathbf{k}}}\right)_{k=k_{F}}
$$

where $n_{F}(x)=\left(e^{\beta x}+1\right)^{-1}$ with $\beta=1 / T$ and $E_{\mathbf{k}}=$ $\sqrt{\epsilon_{\mathbf{k}}^{2}+\left|\Delta_{\mathbf{k}}\right|^{2}}$ is the quasiparticle energy with normal state dispersion $\epsilon_{\mathbf{k}}=\frac{k^{2}}{2 m}-\mu_{F}$. We compute the anisotropic contributions from, with $\omega_{n}=\pi T(2 n+1)$,

$$
\begin{aligned}
\int_{-\infty}^{\infty} d \epsilon\left(-\frac{\partial n_{F}}{\partial E_{\mathbf{k}}}\right)_{k_{F}} & =\int_{-\infty}^{\infty} d \epsilon T \sum_{n=-\infty}^{\infty} \frac{\omega_{n}^{2}-E_{k_{F}}^{2}}{\left(\omega_{n}^{2}+E_{k_{F}}\right)^{2}} \\
& =\pi T \sum_{n=-\infty}^{\infty} \frac{-\left|\Delta_{k_{F}}\right|^{2}}{\left(\omega_{n}^{2}+\left|\Delta_{k_{F}}\right|^{2}\right)^{3 / 2}}
\end{aligned}
$$

The dimensionless summation variable is $x_{n}=\frac{\pi T}{\Delta_{0}}(2 n+1)$, giving for $T \ll \Delta_{0}$

$$
\begin{aligned}
\frac{\rho_{n \|}^{(0)}}{3 \rho} & =\frac{\pi T}{\Delta_{0}} \sum_{n=-\infty}^{\infty} \int_{-1}^{1} d u \frac{-u^{2}\left(1-u^{2}\right)}{\left(x_{n}^{2}+\left(1-u^{2}\right)\right)^{3 / 2}} \\
& =\frac{\pi T}{2 \Delta_{0}} \sum_{n=-\infty}^{\infty}\left[3\left|x_{n}\right|-\left(3 x_{n}^{2}+1\right) \arctan \left(\frac{1}{\left|x_{n}\right|}\right)\right] \\
& =\frac{\pi T}{\Delta_{0}} \sum_{n=-\infty}^{\infty}\left[4\left|x_{n}\right|-\left(3 x_{n}^{2}-1\right) \frac{\pi}{2}+O\left(x_{n}^{4}\right)\right]
\end{aligned}
$$

and

$$
\begin{aligned}
\frac{\rho_{n \perp}^{(0)}}{3 \rho} & =\frac{\pi T}{\Delta_{0}} \sum_{n=-\infty}^{\infty} \int_{-1}^{1} \frac{d u}{4} \frac{-\left(1-u^{2}\right)^{2}}{\left(x_{n}^{2}+\left(1-u^{2}\right)\right)^{3 / 2}} \\
& =\frac{\pi T}{4 \Delta_{0}} \sum_{n=-\infty}^{\infty}\left[3\left|x_{n}\right|-\frac{2\left|x_{n}\right|}{1+x_{n}^{2}}+\left(1-3 x_{n}^{2}\right) \arctan \left(\frac{1}{\left|x_{n}\right|}\right)\right] \\
& =\frac{\pi T}{4 \Delta_{0}} \sum_{n=-\infty}^{\infty}\left[-\frac{16}{3}\left|x_{n}\right|^{3}+\left(3 x_{n}^{2}-1\right) \frac{\pi}{2}+O\left(x_{n}^{5}\right)\right] .
\end{aligned}
$$

We use the regularizations, $\zeta_{a}(s)=\sum_{n=0}^{\infty}(n+a)^{s}$,

$$
\begin{gathered}
\sum_{n=-\infty}^{\infty}=1+2 \sum_{n=1}^{\infty}=1+2 \zeta(0)=0, \\
\sum_{n=-\infty}^{\infty}\left|x_{n}\right|=\frac{4 \pi T}{\Delta_{0}} \zeta_{1 / 2}(-1)=\frac{\pi T}{6 \Delta_{0}}, \\
\sum_{n=-\infty}^{\infty} x_{n}^{2}=\frac{8 \pi^{2} T^{2}}{\Delta_{0}^{2}} \zeta_{1 / 2}(-2)=0, \\
\sum_{n=-\infty}^{\infty}\left|x_{n}\right|^{3}=\frac{16 \pi^{3} T^{3}}{\Delta_{0}^{3}} \zeta_{1 / 2}(-3)=-\frac{7 \pi^{3} T^{3}}{60 \Delta_{0}^{3}} .
\end{gathered}
$$

These give the result in Eq. (26). Similarly $\rho_{s i j}(T)=\rho \delta_{i j}-$ $\rho_{n i j}(T)$ by Galilean invariance, valid at all temperatures with or without Fermi liquid corrections.

From the superfluid and normal densities, the conclusion is that only anisotropic momenta of the order of $\Delta p_{\perp} \sim \frac{T}{\Delta_{0}} p_{F}=$ $\frac{T}{c_{\perp}}$ and $\Delta p_{\|}=\left(\frac{c_{\perp}}{c_{\|}}\right) \frac{T}{c_{\perp}}, c_{\|}=v_{F}$ contribute in the vicinity of the gap nodes to the thermal fluctuations $\epsilon \sim T$.

\section{Quasirelativistic chiral fermions with torsion a. Anisotropic Landau level model}

The quasirelativistic model for the chiral superfluid with anisotropic dispersion is, with $\hat{\mathbf{l}} \simeq \hat{\mathbf{z}}$,

$$
\epsilon_{p}=\frac{p^{2}}{2 m}-\mu_{F} \rightarrow \epsilon_{p_{z}} \equiv \frac{p_{z}^{2}}{2 m}-\mu_{F},
$$

which is valid for real ${ }^{3} \mathrm{He}-\mathrm{A}$ for $E \ll m c_{\perp}^{2}, c_{\perp} \equiv \Delta_{0} / p_{F}$. We approximate $\epsilon(\mathbf{p})=\frac{p^{2}-p_{F}^{2}}{2 m} \simeq \frac{p_{z}^{2}-p_{F}^{2}}{2 m}=\epsilon\left(p_{z}\right)$, strictly valid when $p_{\perp} \ll\left(\frac{c_{\perp}}{c_{\|}}\right) p_{F}, p_{\|} \equiv p_{z} \ll\left(\frac{c_{\perp}}{c_{\|}}\right)^{2} p_{F}$ which coincides with the linear Weyl regime $\epsilon_{p_{z}}= \pm v_{F}\left(p_{z}-p_{F}\right)$. However, we can fix the anisotropic dispersion to $\epsilon\left(p_{z}\right)$ for all momenta to obtain a convenient UV-complete model. The following analysis for the total vacuum current is valid, as long as the correct cutoff for the linear or anisotropic Weyl regime is maintained for ${ }^{3} \mathrm{He}-\mathrm{A}$, see Ref. [36].

In particular for the case of the anomalous current, only the momentum along $\hat{\mathbf{l}} \simeq \hat{\mathbf{z}}$ contributes justifying the approximation to the dispersion and the Landau level (LL) model. Actually the leading contribution is from curvature [16] perpendicular to $\hat{\mathbf{l}}$, requiring the anisotropic cutoffs below $E_{\text {Weyl }}$ in the LL model, discussed already. The results generalizes otherwise by the NY form (2). Similarly, for thermal fluctuations in the LL model, we get the correct contributions, as long 
as the cutoff $T \ll \Delta_{0}$ and $E \ll m c_{\perp}^{2}=\frac{c_{\perp}}{c_{\|}} \Delta_{0}$ are maintained. In particular, this requires that

$$
\begin{gathered}
\epsilon_{p}\left(p_{F}+\Delta p\right) \rightarrow \epsilon_{p_{z}}\left(p_{F}+\Delta p_{\|}\right) \\
\sim c_{\|}\left(\frac{c_{\perp}}{c_{\|}}\right) \frac{T}{2 c_{\perp}} \sim \frac{T}{2} .
\end{gathered}
$$

That is $\Delta p_{\perp} \sim \frac{T}{\Delta_{0}} p_{F}=\frac{T}{c_{\perp}}, \Delta p_{\|}=\left(\frac{c_{\perp}}{c_{\|}}\right) \frac{T}{c_{\perp}}$ for both $T, \epsilon \ll$ $m c_{\perp}^{2}$. This follow also by the thermal equipartition principle corresponding to the superfluid results in Appendix A 1. For the anomaly, only the gapless chiral lowest LLs are relevant, while the gapped levels, with gap $\sim c_{\perp} \sqrt{p_{F} T_{B}}$ are particlehole symmetric and cancel out $[39,45]$. The dispersion of the lowest LL becomes $E_{n=0}=-\operatorname{sgn}\left(p_{z} T_{B}\right) \epsilon\left(p_{z}\right)$. The density of state per $p_{z}$ per Landau level is

$$
N_{\mathrm{LL}}\left(p_{z}\right)=\frac{\left|p_{z} T_{B}\right|}{4 \pi^{2}},
$$

where $T_{B}=\hat{\mathbf{l}} \cdot \nabla \times \hat{\mathbf{l}}$. The lowest LL is particlelike (holelike) for $p_{W}=\mp p_{F} \hat{\mathbf{l}}$. For more on this anisotropic chiral fermion with torsion and the corresponding Newton-Cartan space-time, see Ref. [16] and the forthcoming paper with the anisotropic LL model [36].

\section{b. Vacuum currents}

The quasirelativistic integrals for the chiral currents (16),(20) in the main text are given by the lowest Landau level (LLL) contribution, which is particle- and holelike for $p_{z}<0$ and $p_{z}>0$, respectively. In the chiral superfluid, the quasiparticles are spin-degenerate Majorana-Weyl excitations giving a compensating factor of two. It follows that the quasiparticle momentum along the $\hat{\mathbf{l}} \approx \hat{\mathbf{z}}$ direction is, with the quasiparticle distribution function $n_{F}(x)=\left(e^{\beta x}+1\right)^{-1}$,

$$
\begin{aligned}
\mathbf{P}_{\mathrm{qp}} \cdot \hat{\mathbf{l}} & =-2 \int_{0}^{\infty} N_{\mathrm{LL}}\left(p_{z}\right) d p_{z} p_{z} n_{F}\left(\epsilon_{p_{z}}-\mu_{F}\right) \\
& =-\frac{2 T_{B}}{4 \pi^{2}} \int_{0}^{\infty} d p_{z} p_{z}^{2} n_{F}\left(\epsilon_{p_{z}}-\mu_{F}\right) \\
& =-\frac{T_{B}}{2 \pi^{2}}\left[\int_{0}^{p_{F}} d p_{z} p_{z}^{2}+\frac{4 p_{F}}{c_{\perp}^{2}} \int_{0}^{\infty} d x x n_{F}(x)\right] \\
& =-\left(\frac{p_{F}^{3}}{6 \pi^{2}}+\frac{p_{F} T^{2}}{6 c_{\perp}^{2}}\right) \hat{\mathbf{l}} \cdot \nabla \times \hat{\mathbf{I}} \\
& =\mathbf{j}_{\text {anom } \|}^{\mathrm{vac}}+\mathbf{j}_{\text {anom } \|}^{\mathrm{qp}}(T),
\end{aligned}
$$

where we have used $T \ll \Delta \ll \mu_{F}$ and the linearization of the spectrum close to $\mu_{F}$ with the anisotropic scaling (A7) which applies for (weak coupling) ${ }^{3} \mathrm{He}-\mathrm{A}$. The vacuum contribution is

$$
\mathbf{j}_{\mathrm{anom} \|}^{\mathrm{vac}}=-\frac{T_{B}}{2 \pi^{2}} \int_{0}^{\infty} d p_{z} p_{z}^{2} \Theta\left(p_{F}-p_{z}\right),
$$

whereas the quasiparticle current due to the thermal fluctuations is

$$
\mathbf{j}_{\mathrm{anom} \|}^{\mathrm{qp}}(T)=-\frac{p_{F} T^{2}}{6 c_{\perp}^{2}} \hat{\mathbf{l}}(\hat{\mathbf{l}} \cdot \nabla \times \hat{\mathbf{l}}) .
$$

Similarly, the thermal density of states is the vicinity of the nodes for $T \ll \Delta_{0}$ is

$$
\begin{aligned}
n_{5}(T) & =-2 \int_{0}^{\infty} N_{\mathrm{LL}}\left(p_{z}\right) d p_{z}\left[n_{F}\left(\epsilon_{p_{z}}-\mu_{F}\right)-\Theta\left(\mu-\epsilon_{p_{z}}\right)\right] \\
& =-\frac{T_{B}}{2 \pi^{2}} \int_{0}^{\infty} d x x n_{F}(x) \\
& =-\frac{T^{2}}{12 c_{\perp}^{2}}(\hat{\mathbf{l}} \cdot \nabla \times \hat{\mathbf{l}}),
\end{aligned}
$$

where the $T=0$ vacuum contribution is subtracted, since only contribution from the vicinity of the nodes to the chiral density is well-defined. The relevant thermal integral is similar to Eq. (A2)

$$
\begin{gathered}
\int_{0}^{\infty} d x x_{F}(x)=\frac{1}{4} \int_{-\infty}^{\infty} x^{2}\left(-\frac{\partial n_{F}(x)}{\partial x}\right) \\
=4 \pi^{2} T^{2} \zeta_{1 / 2}(-1)=\frac{\pi^{2} T^{2}}{6} .
\end{gathered}
$$

\section{Relativistic chiral fermions}

Now we compare these results with relativistic Weyl fermions with both positive and negative energy branches, i.e., particles and antiparticles with the node at $p_{z}=0$. At chemical potential $\mu$ at the node, the corresponding momentum along $\mathbf{I}$ is

$$
\begin{aligned}
\mathbf{P}_{\mathrm{W}} \cdot \hat{\mathbf{I}} & =\int_{-\infty}^{\infty} N_{\mathrm{LL}}\left(p_{z}\right) d p_{z} p_{z}\left[n_{F}\left(\epsilon_{p_{z}}-\mu\right)-\Theta\left(-p_{z}\right)\right] \\
& =-\frac{T_{B}}{4 \pi^{2}} \int_{0}^{\infty} d p_{z} p_{z}^{2}\left[n_{F}\left(\epsilon_{p_{z}}-\mu\right)-n_{F}\left(\epsilon_{p_{z}}+\mu\right)\right] \\
& =-\frac{T_{B}}{4 \pi^{2}}\left[\int_{0}^{\mu} d p_{z} p_{z}^{2}+4 \mu \int_{0}^{\infty} d x x n_{F}\left(\frac{x}{T}\right)\right] \\
& =-\left(\frac{\mu^{3}}{12 \pi^{2}}+\frac{\mu T^{2}}{12}\right) \hat{\mathbf{l}} \cdot \nabla \times \hat{\mathbf{l}},
\end{aligned}
$$

per single node, in units where $\epsilon_{p_{z}}=p_{z}$. The thermal chiral density, corresponding to the left-handed node,

$$
\begin{aligned}
n_{W-}(T) & =-\int_{-\infty}^{\infty} N_{\mathrm{LL}}\left(p_{z}\right) d p_{z}\left[n_{F}\left(\epsilon_{p_{z}}-\mu\right)-\Theta\left(-p_{z}\right)\right] \\
& =-\frac{T_{B}}{4 \pi^{2}} \int_{0}^{\infty} d p_{z} p_{z}\left[n_{F}\left(\epsilon_{p_{z}}-\mu\right)+n_{F}\left(\epsilon_{p_{z}}+\mu\right)\right] \\
& =\left(-\frac{\mu^{2}}{8 \pi^{2}}-\frac{T^{2}}{24}\right) \hat{\mathbf{l}} \cdot \nabla \times \hat{\mathbf{l}} .
\end{aligned}
$$

In order to compute the integral, we have used $n_{F}(x)=1-$ $n_{F}(-x)$ as well as the linear expansion close to the nodes for $T \ll \Delta_{0}$. Intriguingly, the structure of this momentumdensity and density contribution are exactly of the same form $[28,30,32,37,38]$. The difference is, of course, that $\mu$ is counted from the node and has different sign for positive energy solutions corresponding to the antiparticles and that the contribution of filled vacuum states is subtracted. Lastly, we note the dissimilarity of these relativistic finite chiral chemical potential and temperature terms to Eqs. (32)-(34). 
[1] G. E. Volovik, The Universe in a Helium Droplet (Clarendon Press, Oxford, 2003).

[2] P. Horava, Stability of Fermi Surfaces and $K$ Theory, Phys. Rev. Lett. 95, 016405 (2005).

[3] C. Herring, Accidental degeneracy in the energy bands of crystals, Phys. Rev. 52, 365 (1937).

[4] A. A. Abrikosov and S. D. Beneslavskii, Possible existence of substances intermediate between metals and dielectrics, ZhETF 59, 1280 (1970) [JETP 32, 699 (1971)].

[5] H. B. Nielsen and M. Ninomiya, The Adler-Bell-Jackiw anomaly and Weyl fermions In a crystal, Phys. Lett. B 130, 389 (1983).

[6] G. E. Volovik, Chiral anomaly and the law of conservation of momentum in ${ }^{3} \mathrm{He}-\mathrm{A}$, Pis'ma ZhETF 43, 428 (1986); [JETP Lett. 43, 551 (1986)].

[7] A. A. Zyuzin and A. A. Burkov, Topological response in Weyl semimetals and the chiral anomaly, Phys. Rev. B 86, 115133 (2012).

[8] H. T. Nieh and M. L. Yan, An identity In Riemann-Cartan geometry, J. Math. Phys. 23, 373 (1982).

[9] H. T. Nieh and M. L. Yan, Quantized Dirac field in curved Riemann-Cartan background. I. Symmetry properties, Green's function, Ann. Phys. 138, 237 (1982).

[10] H. T. Nieh, A torsional topological invariant, Int. J. Mod. Phys. A 22, 5237 (2007).

[11] S. Yajima, Evaluation of the heat kernel in Riemann-Cartan space, Class. Quantum Grav. 13, 2423 (1996).

[12] Y. N. Obukhov, E. W. Mielke, J. Budczies, and F. W. Hehl, On the chiral anomaly in non-Riemannian space-times, Found. Phys. 27, 1221 (1997).

[13] O. Chandia and J. Zanelli, Topological invariants, instantons, and the chiral anomaly on spaces with torsion, Phys. Rev. D 55, 7580 (1997).

[14] O. Chand \pm a and J. Zanelli, Torsional topological invariants (and their relevance for real life), AIP Conf. Proc. 419, 251 (1998).

[15] O. Chandia and J. Zanelli, Supersymmetric particle in a spacetime with torsion and the index theorem, Phys. Rev. D 58, 045014 (1998).

[16] J. Nissinen, Emergent Space-time and Gravitational Nieh-Yan Anomaly in Chiral $p+i p$ Weyl Superfluids and Superconductors, Phys. Rev. Lett. 124, 117002 (2020).

[17] O. Parrikar, T. L. Hughes, R. G. Leigh, Torsion, parity-odd response and anomalies in topological states, Phys. Rev. D 90, 105004 (2014).

[18] Y. Ferreiros, Y. Kedem, E. J. Bergholtz, and J. H. Bardarson, Mixed Axial-Torsional Anomaly in Weyl Semimetals, Phys. Rev. Lett. 122, 056601 (2019).

[19] G. E. Volovik and A. I. Zelnikov, Universal temperature corrections to the free energy for the gravitational field, Pisma ZhETF 78, 1271 (2003); [JETP Lett. 78, 751 (2003)].

[20] L. Sun and S. Wan, Chiral viscoelastic response in Weyl semimetals, Europhys. Lett. 108, 37007 (2014).

[21] D. T. Son, Newton-Cartan geometry and the quantum Hall effect, arXiv:1306.0638.

[22] G. E. Volovik, The gravitational-topological Chern-Simons term in a film of superfluid ${ }^{3} \mathrm{He}-\mathrm{A}$, Pis'ma ZhETF 51, 111 (1990); [JETP Lett. 51, 125 (1990)].

[23] N. Read, D. Green, Paired states of fermions in two dimensions with breaking of parity and time reversal symmetries and the fractional quantum Hall effect, Phys. Rev. B 61, 10267 (2000).

[24] X.-Y. Song, Y.-C. He, Ashvin Vishwanath, and Chong Wang, Electric polarization as a nonquantized topological response and boundary Luttinger theorem, arXiv:1909.08637.

[25] J. Nissinen and G. E. Volovik, Tetrads in solids: from elasticity theory to topological quantum Hall systems and Weyl fermions, ZhETF 154, 1051 (2018); [JETP 127, 948 (2018)].

[26] J. Nissinen and G. E. Volovik, Elasticity tetrads, mixed axialgravitational anomalies, and (3+1)-d quantum Hall effect, Phys. Rev. Research 1, 023007 (2019).

[27] G. E. Volovik and A. Vilenkin, Macroscopic parity violating effects and ${ }^{3}$ He-A, Phys. Rev. D 62, 025014 (2000).

[28] G. Başar, D. E. Kharzeev, and H.-U. Yee, Triangle anomaly in Weyl semimetals, Phys. Rev. B 89, 035142 (2014).

[29] K. Landsteiner, E. Megías, and F. Pena-Benitez, Gravitational Anomaly and Transport Phenomena, Phys. Rev. Lett. 107, 021601 (2011).

[30] R. Loganayagam, P. Surówka, Anomaly/transport in an ideal Weyl gas, J. High Energy Phys. 04 (2012) 097.

[31] K. Jensen, R. Loganayagam, and A. Yarom, Thermodynamics, gravitational anomalies and cones, J. High Energ. Phys. 02 (2013) 088 .

[32] K. Landsteiner, Anomalous transport of Weyl fermions in Weyl semimetals, Phys. Rev. B 89, 075124 (2014).

[33] A. Lucas, R. A. Davison, and S. Sachdev, Hydrodynamic theory of thermoelectric transport and negative magnetoresistance in Weyl semimetals, Proc. Natl. Acad. Sci. USA 113, 9463 (2016).

[34] M. Stone, Y. Kim, Mixed anomalies: Chiral vortical effect and the Sommerfeld expansion, Phys. Rev. D 98, 025012 (2018).

[35] J. Gooth, A. C. Niemann, T. Meng, A. G. Grushin, K. Landsteiner, B. Gotsmann, F. Menges, M. Schmidt, C. Shekhar, V. Süss, R. Hühne, B. Rellinghaus, C. Felser, Binghai Yan, K. Nielsch, Experimental signatures of the mixed axialgravitational anomaly in the Weyl semimetal $\mathrm{NbP}$, Nature (London) 547, 324 (2017).

[36] S. Laurila and J. Nissinen, Torsional Landau levels and geometric anomalies in condensed matter Weyl systems, arXiv:2007.10682.

[37] Z. V. Khaidukov and M. A. Zubkov, Chiral torsional effect, Pis'ma ZhETF 108, 702 (2018); [JETP Lett. 108, 670 (2018)].

[38] S. Imaki and A. Yamamoto, Lattice field theory with torsion, Phys. Rev. D 100, 054509 (2019).

[39] G. E. Volovik, Normal Fermi liquid in a superfluid ${ }^{3} \mathrm{He}-\mathrm{A}$ at $T=0$ and the anomalous current, Pis'ma ZhETF 42, 294 (1985) [JETP Lett. 42, 363 (1985)].

[40] Z.-M. Huang, B. Han, and M. Stone, The Nieh-Yan anomaly: torsional Landau levels, central charge and anomalous thermal Hall effect, Phys. Rev. B 101, 125201 (2020).

[41] T. Kobayashi, T. Matsushita, T. Mizushima, A. Tsuruta, and S. Fujimoto, Negative Thermal Magnetoresistivity as A Signature of a Chiral Anomaly in Weyl Superconductors, Phys. Rev. Lett. 121, 207002 (2018).

[42] A. Flachi and V. Vitagliano, Symmetry breaking and lattice kirigami: Finite temperature effects, Phys. Rev. D 99, 125010 (2019).

[43] K. Landsteiner, Notes on Anomaly Induced Transport, Acta Phys. Pol. B 47, 2617 (2016). 
[44] R. Ilan, A. G. Grushin, and D. I. Pikulin, Pseudoelectromagnetic fields in 3D topological semimetals, Nat. Rev. Phys. 2, 29 (2020).

[45] A. V. Balatskii, G. E. Volovik, and V. A. Konyshev, On the chiral anomaly in superfluid ${ }^{3} \mathrm{He}-\mathrm{A}$, ZhETF 90, 2038 (1986); [JETP 63, 1194 (1986)].

[46] D. Vollhardt and P. Wölfle, The Superfluid Phases of Helium 3 (Taylor \& Francis, London, 1990).

[47] M. C. Cross, A generalized Ginzburg-Landau approach to the superfluidity of helium 3, J. Low Temp. Phys. 21, 525 (1975).

[48] G. E. Volovik and V. P. Mineev, Orbital angular momentum and orbital dynamics: 3He-A and the Bose liquid, ZhETF 81, 989 (1981); [JETP 54, 524 (1981)].

[49] G. E. Volovik, Axial anomaly in 3He-A: Simulation of baryogenesis and generation of primordial magnetic field in Manchester and Helsinki, Physica B 255, 86 (1998).

[50] D. Diakonov, Towards lattice-regularized quantum gravity, arXiv:1109.0091.

[51] Y. N. Obukhov, F. W. Hehl, Extended Einstein-Cartan theory à la Diakonov: The field equations, Phys. Lett. B 713, 321 (2012).

[52] B. Bradlyn and N. Read, Low-energy effective theory in the bulk for transport in a topological phase, Phys. Rev. B 91, 125303 (2015).

[53] A. Gromov and A. G. Abanov, Thermal Hall Effect and Geometry with Torsion, Phys. Rev. Lett. 114, 016802 (2015).
[54] C. Copetti, Torsion and anomalies in the warped limit of Lifschitz theories, J. High Energy Phys. 01 (2020) 190.

[55] Z.-M. Huang, B. Han, and M. Stone, Hamiltonian approach to the torsional anomalies and its dimensional ladder, Phys. Rev. B 101, 165201 (2020).

[56] H. Shapourian, T. L. Hughes, and S. Ryu, Viscoelastic response of topological tight-binding models in two and three dimensions, Phys. Rev. B 92, 165131 (2015).

[57] J. M. Luttinger, Theory of thermal transport coefficients, Phys. Rev. 135, A1505 (1964).

[58] G. E. Volovik, Wess-Zumino action for the orbital dynamics of ${ }^{3} \mathrm{He}-\mathrm{A}$, Pis'ma ZhETF 44, 144 (1986); [JETP Lett. 44, 185 (1986).

[59] R. Combescot and T. Dombre, Twisting in superfluid ${ }^{3} \mathrm{He}-\mathrm{A}$ and consequences for hydrodynamics at $T=0$, Phys. Rev. B 33, 79 (1986).

[60] L. Liang and Teemu Ojanen, Topological magnetotorsional effect in Weyl semimetals, Phys. Rev. Research 2, 022016(R) (2020).

[61] J. Nissinen and G. E. Volovik, On thermal Nieh-Yan anomaly in topological Weyl materials, Pis'ma ZhETF 110, 797 (2019); JETP Lett. 110, 789 (2019).

[62] S. Imaki and Zebin Qiu, Chiral torsional effect with finite temperature, density and curvature, Phys. Rev. D 102, 016001 (2020). 